\title{
Development of a high-speed rail transmission system using digital signal processors for railway signalling
}

\author{
H. Mochizuki ${ }^{1}$, S. Takahashi ${ }^{1}$, H. Nakamura ${ }^{1}$, S. Nishida ${ }^{2}$ \\ \& R. Ishikawa ${ }^{2}$ \\ ${ }^{1}$ Department of Electronics and Computer Science, \\ Nihon University, Japan \\ ${ }^{2}$ Kyosan Electric Mfg. Co., Ltd., Japan
}

\begin{abstract}
Railway signalling systems that transmit control information via rails have been deployed in many applications, for example, digital automatic train control (ATC) systems for controlling train speed. Since the performance of digital ATC systems depends on the signal transmission speed, recently there have been many studies aimed at realizing high-speed data transmission. However, it is difficult to increase the transmission speed because rails have strong attenuation at frequencies above about $10 \mathrm{kHz}$. Therefore, the present transmission speed limit of digital ATC using rails is about 300 bps. To overcome these limitations, we aimed to increase the transmission speed by improving the modulation method. In this paper, we propose a new modulation method combining codedivision multiple access (CDMA) and quadrature amplitude modulation (QAM), called the CDMA-QAM method. This method transmits QAM symbols allocated based on a CDMA signal. It has the advantage that QAM errors can be recovered if they are in a range where they can be absorbed by CDMA demodulation. We developed a CDMA-QAM rail transmission system based on digital signal processors. We conducted a field experiment using an actual rail and verified that the system can achieve favourable constellation characteristics with about 20 $\mathrm{dB}$ attenuation of the received signal at a carrier frequency of $3 \mathrm{kHz}$.
\end{abstract}

Keywords: railway signalling, code-division multiple access, quadrature amplitude modulation, digital signal processor. 


\section{Introduction}

Railway signalling systems that transmit information via rails have been deployed in many applications, for example, digital automatic train control (ATC) systems for controlling train speed. Since the performance of digital ATC systems depends on the transmission speed, recently there has been a great deal of research aimed at realizing high-speed data transmission [1]. Using the rail as a transmission medium has several benefits, such as superior security and cost performance compared to wireless communication methods. However, it is difficult to increase the transmission speed because rails exhibit strong attenuation at frequencies above about $10 \mathrm{kHz}$. Therefore, the present transmission speed of digital ATC using rails is limited to about $300 \mathrm{bps}$. To overcome this restriction, we aimed to increase the transmission speed by improving the modulation method.

\section{CDMA-QAM rail transmission system}

\subsection{Overview of the CDMA-QAM method}

We propose a new modulation method combining code-division multiple access (CDMA) and quadrature amplitude modulation (QAM), called the CDMA-QAM method. Figure 1 is a block diagram schematically illustrating the CDMA-QAM method.

The CDMA-QAM method transmits QAM symbols allocated based on a multiplexed CDMA signal. Specifically, the transmitter first divides the transmit data into 63 parallel data channels. The multiplexed CDMA signal is generated by adding all channels after subjecting them to CDMA modulation. Finally, the multiplexed signal is allocated to 64QAM symbols according to the amplitude of the multiplexed signal, from 0 to 63 , and is transmitted on the transmission line. After the amplitude of the multiplexed signal is converted into binary, the three higher-order bits are allocated to an I (in phase) channel, and the three lowerorder bits are allocated to a Q (quadrature) channel. Figure 2 shows the amplitude data allocation of each symbol in the CDMA-QAM method.

At the receiver, after demodulating the 64QAM and CDMA signals, the parallel data is converted into the receive data. One benefit of the proposed method is that, even if the QAM demodulation has errors, they can be reduced by CDMA. Since QAM does not allocate the transmit data directly, but the multiplexed CDMA signal, QAM errors can be recovered if they are in a range that can be absorbed by the CDMA demodulation.

Next, we propose a symbol allocation scheme for the CDMA-QAM method. Figure 3 shows the results of analysis of the frequency distribution of the multiplexed CDMA signal amplitude in the transmitter. This figure shows that the frequency distribution is not a uniform distribution but is like a normalized distributioncentred on 32 (almost the median value of the multiplexed signal amplitude). This means that the QAM symbols are centred near the Q-axis. On the other hand, the I channel is more important than the $\mathrm{Q}$ channel for the bit 
error rate (BER) characteristics. This is because the I channel is allocated the three higher-order bits of the multiplexed CDMA signal. Based on these facts, we designed a symbol allocation scheme in which the symbol interval of the I channel is wider than that of the $\mathrm{Q}$ channel, as shown in Figure 4. With this scheme, the CDMA-QAM method can improve the BER characteristics without substantially increasing the total electrical power consumption of the system.



Figure 1: $\quad$ Block diagram of the CDMA-QAM method.

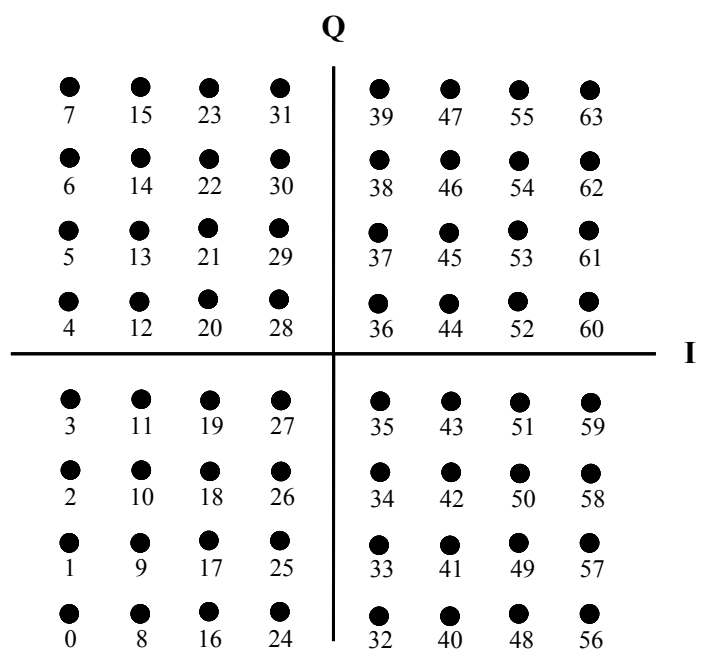

Figure 2: Amplitude data allocation of each symbol in the CDMA-QAM method. 


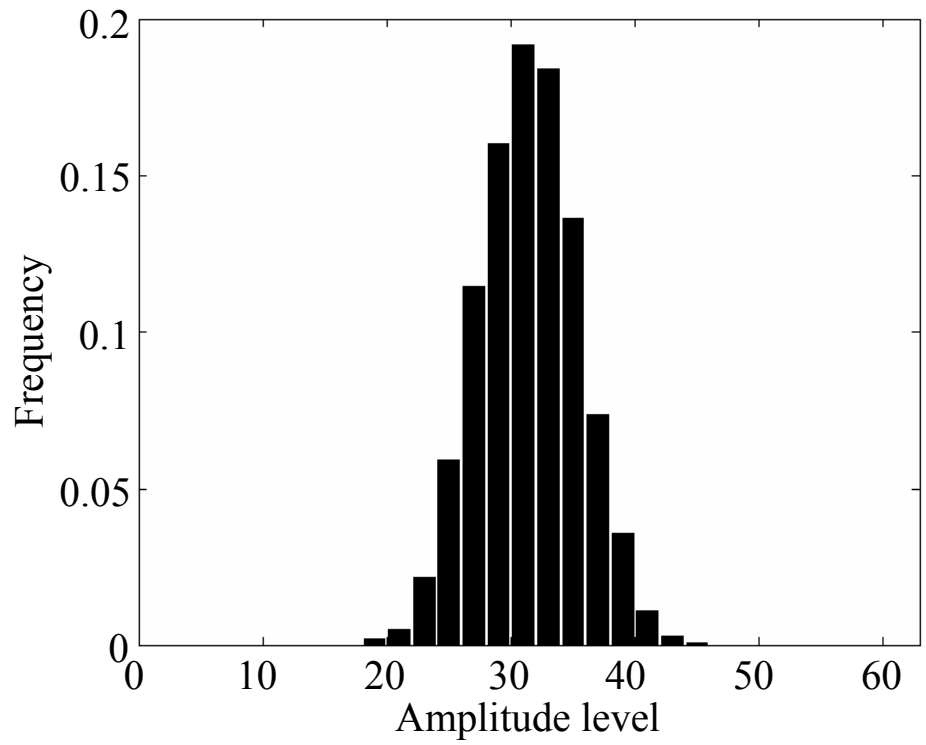

Figure 3: $\quad$ Frequency distribution of multiplexed CDMA signal amplitude.
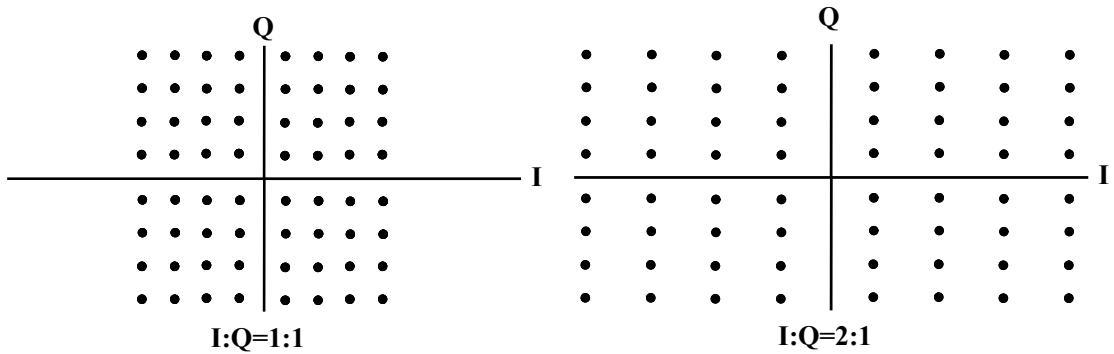

Figure 4: Example of the symbol allocation scheme in the CDMA-QAM method.

\subsection{Evaluation using computer simulation}

We carried out computer simulation in which the I/Q symbol interval rate was varied from $\mathrm{I}: \mathrm{Q}=1: 1$ to $\mathrm{I}: \mathrm{Q}=4: 1$. Figure 5 shows a comparison of the constellation characteristics by $\mathrm{I} / \mathrm{Q}$ symbol interval rate. Here, the characteristic for $I: Q=4: 1$ is normalized with respect to the scale for $I: Q=1: 1$. From this figure, in the characteristic for $\mathrm{I}: \mathrm{Q}=4: 1$, it is possible to reduce the error vector magnitude (EVM) of the I channel, though the EVM of the Q channel is worse than that of $\mathrm{I}: \mathrm{Q}=1: 1$. As mentioned above, since the $\mathrm{I}$ channel is more important than the $Q$ channel for the BER characteristics, $I: Q=4: 1$ is superior. In addition, we evaluated the BER characteristics of our proposed CDMA-QAM rail 
transmission system, and we verified that the BER characteristics improved in proportion to the I/Q symbol interval rate. For example, when the I/Q symbol interval rate was $4: 1$, this system realized a BER of $10^{-5}$, the standard value used in railway signalling, even when the transmission speed was about $2000 \mathrm{bps}$, which is much higher than the transmission speed of current systems [2].
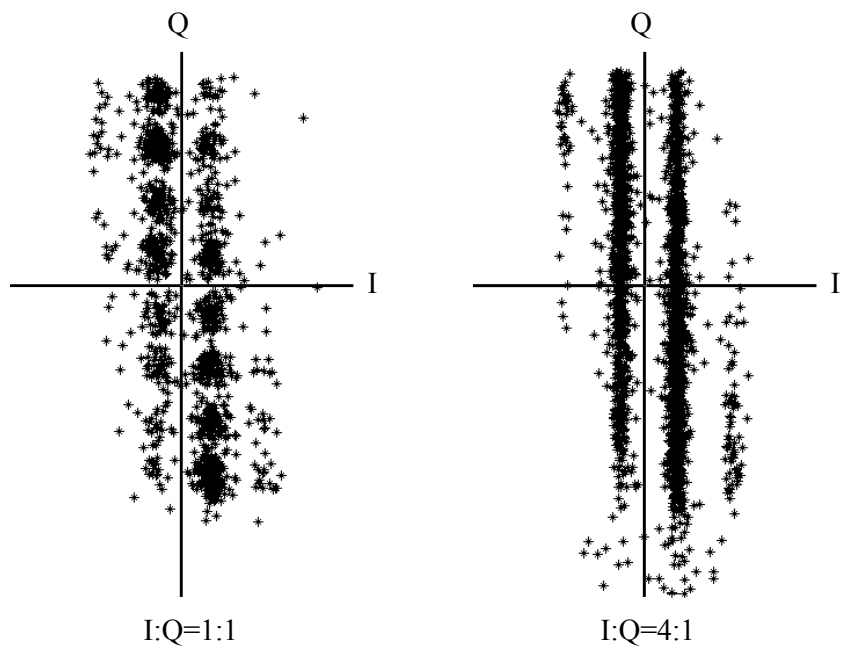

Figure 5: Comparison of constellation characteristics by I/Q symbol interval rate. (The characteristic for $\mathrm{I}: \mathrm{Q}=4: 1$ is normalized by the I-axis.)

\section{Hardware development of the CDMA-QAM transceiver}

\subsection{Hardware architecture}

Figure 6 shows the hardware architecture of the CDMA-QAM transceiver. This figure shows independently developed CDMA and QAM parts. When we compared the amount of processing in the transmitter and receiver, the amount of processing in the receiver was much larger than that in the transmitter because the receiver must perform filtering, synchronous detection, and so on. Therefore, we independently developed the CDMA part and the QAM part in the receiver. We had previously developed the CDMA part. Specifically, we had developed a matched filter that can process all CDMA channels with a field programmable gate array (FPGA) [3]. On the other hand, since this transceiver uses the audiofrequency band, we employed audio digital signal processors (DSPs) in the transmitter and QAM part of the receiver.

\subsection{Hardware development using DSP}

In view of cost, versatility, and so on, we developed the transceiver using a DSP starter kit (DSK). Table 1 shows the specifications of the CDMA-QAM transceiver using DSPs. As mentioned above, since this transceiver uses the 
audio-frequency band, the standard audio interface of the DSPs was used for connection to the rail. In addition, we used general-purpose I/O (GPIO) for the digital interface between the DSP and the CDMA part of the receiver.

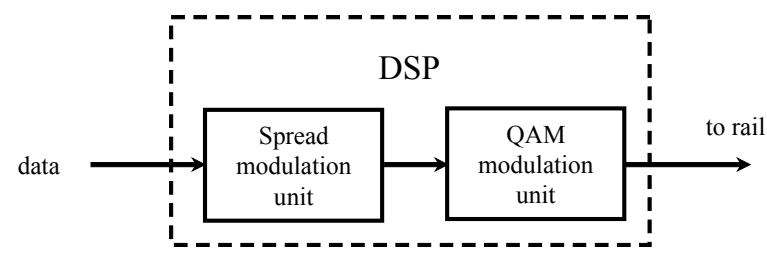

Transmitter

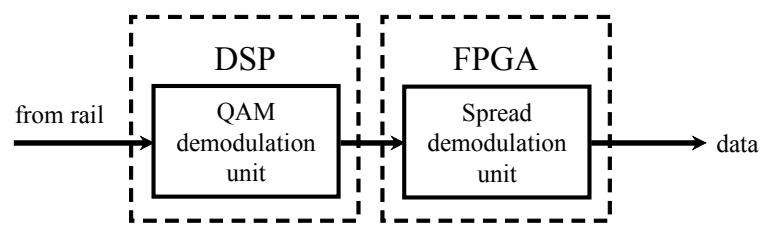

Receiver

Figure 6: Hardware architecture of the CDMA-QAM transceiver.

Table 1: $\quad$ Specifications of the CDMA-QAM transceiver.

\begin{tabular}{|l|l|}
\hline Parameter & Value \\
\hline Carrier frequency & $3000 \mathrm{~Hz}$ \\
\hline Number of multiple access channels & 63 \\
\hline Transmission speed per channel & $23.4375 \mathrm{bps}$ \\
\hline Total transmission speed & $1476.5625 \mathrm{bps}$ \\
\hline Sampling frequency & $48 \mathrm{kHz}$ \\
\hline
\end{tabular}

\subsection{Verification of basic functions}

We conducted an experiment to verify the basic functions of the CDMA-QAM transceiver with the specifications in Table 1, using the setup shown in Figure 7.

As a result, we obtained the CDMA-QAM modulation signal shown in Figure 8, the GPIO digital output shown in Figure 9, and the QAM constellation characteristics shown in Figure 10. As shown in these figures, we verified that this transceiver successfully performed basic functions in real time, such as synchronous detection.

\section{Field experiment using a rail}

We conducted a field experiment using an actual rail based on the setup shown in Figure 11. First we measured the spectral distribution of the CDMA-QAM 


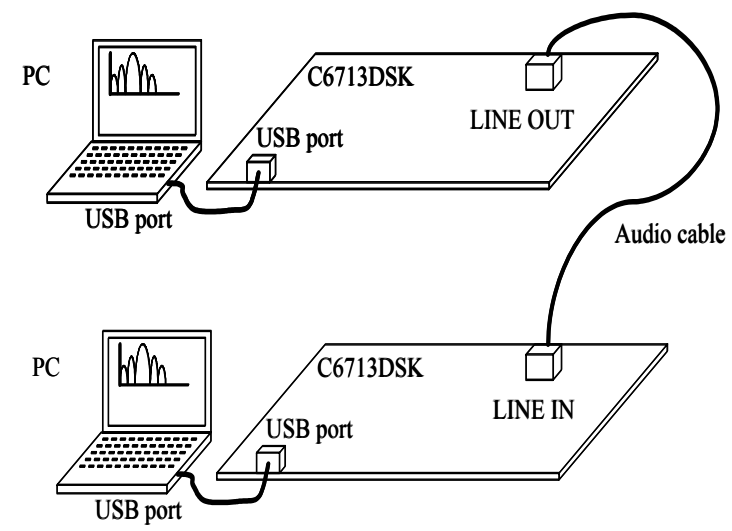

Figure 7: Experimental setup for verifying basic functions.

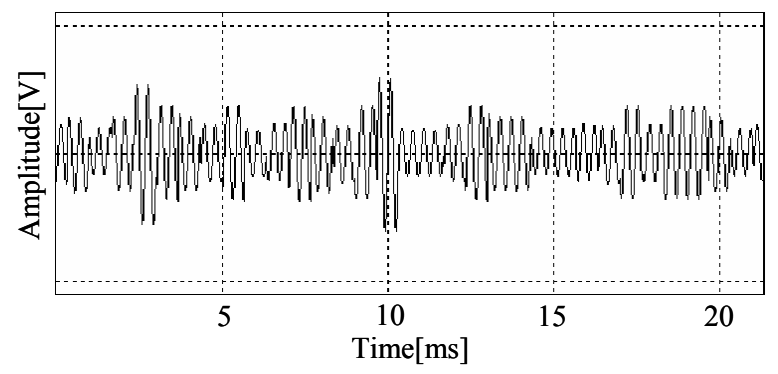

Figure 8: $\quad$ CDMA-QAM modulation signal.

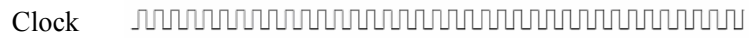

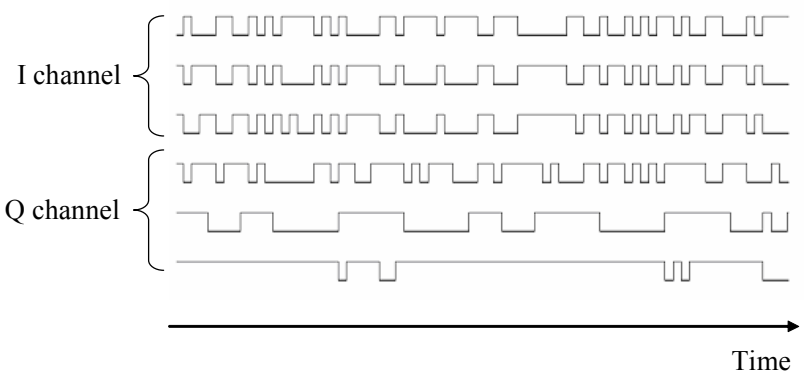

Figure 9: GPIO digital output in the CDMA-QAM receiver.

signal to verify the frequency characteristics of the rail. This result is shown in Figure 12, which shows that the received signal was attenuated by about $20 \mathrm{~dB}$ at the carrier frequency of $3 \mathrm{kHz}$. In view of this characteristic, we employed a software-based automatic gain control (AGC) function in the CDMA-QAM receiver. 


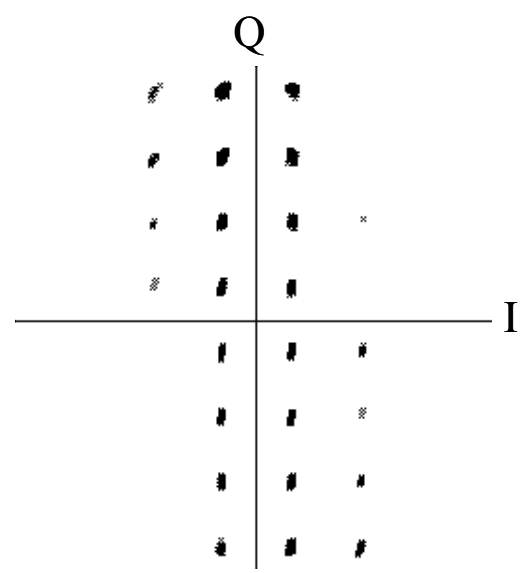

Figure 10: QAM constellation characteristics.

Figure 13 shows constellation characteristics when the train was running. From this figure, we verified that the transceiver achieved suitable constellation characteristics for the I channel. Thus, we could achieve suitable BER characteristics after CDMA demodulation processing.

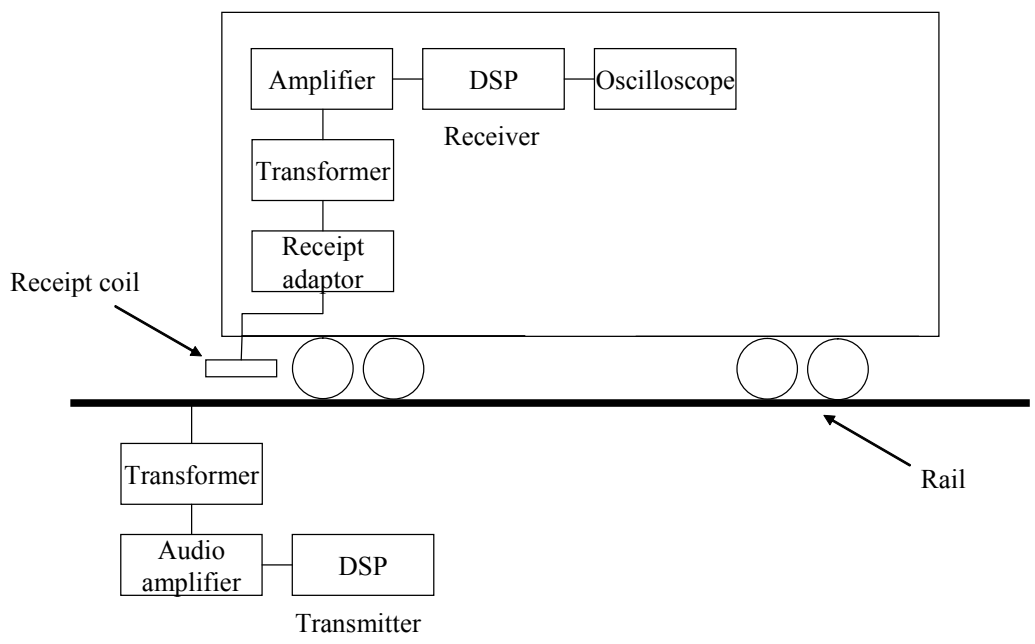

Figure 11: Setup of field experiment using rails.

\section{Conclusion}

In this paper, we proposed a novel CDMA-QAM rail transmission system for improving the transmission speed of rail-based signalling. First we studied the QAM symbol allocation and designed a symbol allocation scheme in which the 

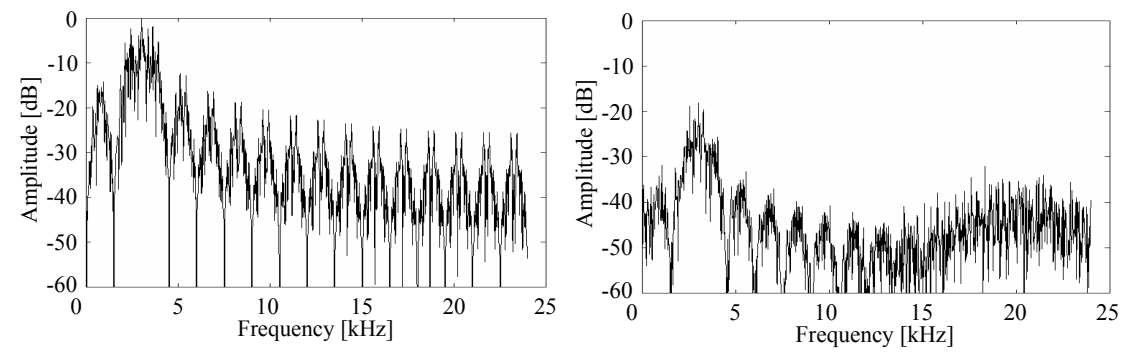

(a) Before signal passed through the rail. (b) After signal passed through the rail.

Figure 12: Spectral distribution at the receiver.

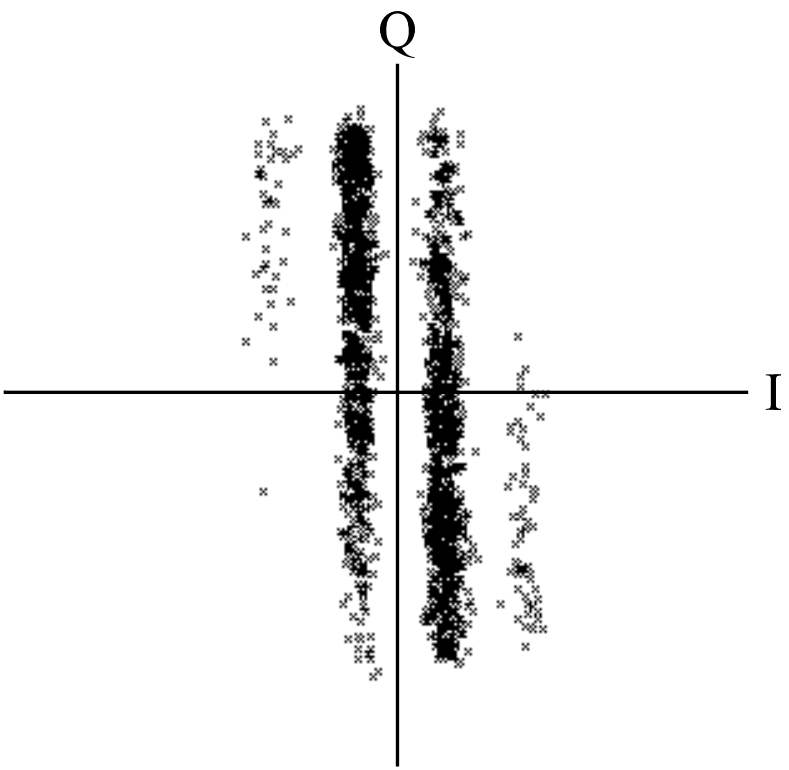

Figure 13: Constellation characteristics for the field experiment.

symbol interval of the I channel is wider than that of the Q channel. We verified that the BER characteristics improved in proportion to the I/Q symbol interval rate.

Next we designed the hardware architecture of the CDMA-QAM rail transmission system and developed a CDMA-QAM transceiver. We verified that this transceiver successfully performed basic functions in real time, such as synchronous detection.

In addition, we conducted a field experiment using an actual rail. We achieved favourable constellation characteristics for the I channel, which is more important for the BER characteristics, with received signal attenuation of about $20 \mathrm{~dB}$ at a carrier frequency of $3 \mathrm{kHz}$. 
In future research, we plan to measure the BER characteristics after combining a CDMA-QAM transceiver with a matched filter, which we have already developed using FPGA, and evaluate the proposed system quantitatively.

\section{Acknowledgements}

This work was supported by Grant-in-Aid for Young Scientists (B), KAKENHI (No. 19760265) from the Japan Society for the Promotion of Science (JSPS), and Nihon University Multidisciplinary Research Grant for 2007.

\section{References}

[1] S. Irie \& T. Hasegawa, "A study on the Railway Signalling System using Spread Spectrum Communication”, IEICE Technical Report, Vol. 93, No. 89, pp. 43-48 (1993-6).

[2] H. Mochizuki, A. Asano, M. Sano, S. Takahashi, \& H. Nakamura, "A Study on Railway Signal System to Apply CDMA-QAM Method", Transactions IEE Japan, Vol. 126-D, No. 3, pp. 345-354 (2006-03).

[3] Y. Sato, H. Mochizuki, S. Takahashi, \& H. Nakamura, "A Development of Matched-filter for Multiple Channel in Railway Signal System Using Spread Spectrum Communication", 2005 Annual Meeting of IEE Japan, 5-189 (2005). 